\title{
Trichophyton mentagrophytes-associated Majocchi's granuloma treated with cryotherapy
}

\author{
Aldona Pietrzak ${ }^{1}$, Krzysztof Tomasiewicz ${ }^{2}$, Jean Kanitakis ${ }^{3}$, Tomasz Paszkowski ${ }^{4}$, \\ Ewa Dybiec ${ }^{5}$, Helena Donica ${ }^{6}$, Agnieszka Wójtowicz ${ }^{7}$, \\ Piotr Terlecki $^{8}$, Grażyna Chodorowska ${ }^{1}$
}

\author{
${ }^{1}$ Chair and Department of Dermatology, Venereology and Pediatric Dermatology, \\ Medical University of Lublin, Poland \\ ${ }^{2}$ Department of Infectious Diseases, Medical University of Lublin, Poland \\ ${ }^{3}$ Department of Dermatology, Ed. Herriot Hospital Group (Pav. R), Lyon, France \\ ${ }^{4} 3^{\text {rd }}$ Department of Gynecology, Medical University of Lublin, Poland \\ ${ }^{5}$ Department of Pediatric Radiology, Medical University of Lublin, Poland \\ ${ }^{6}$ Department of Biochemical Diagnostics, Medical University of Lublin, Poland \\ ${ }^{7}$ Department of Medical Microbiology SPSK1, Lublin, Poland \\ ${ }^{8}$ Department of Vascular Surgery and Angiology, Medical University of Lublin, Poland
}

\begin{abstract}
We here report the case of a woman with dermatophytosis of the thighs due to Trichophyton mentagrophytes where an unusual clinical picture posed considerable diagnostic and therapeutic problems. She presented numerous skin lesions located on the dorsolateral face of the left thigh and the medial surface of the right calf. The initial lesions consisted of small itchy pustules that evolved to exfoliation after scratching. Results of histopathologic examination of a skin biopsy were consistent with dermatophytosis, although the negativity of PAS staining did not allow confirmation of this diagnosis. Direct microscopic examination with $10 \% \mathrm{KOH}$ was negative; however, skin cultures on BioMerieux media revealed Tr. Mentagrophytes. Following the diagnosis of Trichophyton infection, the patient was treated with a combination of isoconazole nitrate and difluocortolone valerate. After therapy, both direct microscopic mycologic examination and culture on BioMerieux medium were negative; however, the lesions persisted, assuming a completely different aspect. Cryotherapy with liquid nitrogen was started. This led to a spectacular improvement: the surface of the skin became almost normal, merely showing slight discoloration. An unusual clinical presentation and non-responsiveness to treatment should prompt revision of the primary diagnosis. A negative result of direct microscopy should not exclude the diagnosis of dermatophytosis. Cryotherapy should be considered in cases that do not respond to conventional antifungal medication. (Folia Histochemica et Cytobiologica 2012, Vol. 50, No. 3, 486-489)
\end{abstract}

Key words: Trichophyton, Majocchi's granuloma, cryotherapy

\section{Introduction}

Dermatophytosis is a common skin infection involving the scalp, glabrous skin and nails. Causative agents

Correspondence address: A. Pietrzak, Chair and Department of Dermatology, Venereology and Pediatric Dermatology,

Medical University of Lublin,

Radziwillowska Str. 13, 20-080 Lublin, Poland;

e-mail: aldonkapietrzak2@o2.pl belong to the closely-related group of fungi known as dermatophytes, which show a unique enzymatic capacity to use keratin as a nutrient source [1]. The clinical manifestations of dermatophytosis are determined by different factors, of which the most important are the particular species involved, the location of the infection, and the status of the host's immune system. Zoophilic dermatophytes induce a stronger host response than do anthropophilic ones. The inflammation is more severe toward Trichophyton (Tr.) mentagrophytes [1-3]. 
Tinea corporis refers to dermatophyte infection of the glabrous skin. It is caused by anthropophilic species such as Tr. rubrum, usually following spreading from another body site, or by geophilic and zoophilic species such as Microsporon (M.) gypseum and $M$. canis following contact with either contaminated soil or an animal host [4-5]. We here report the case of a woman with dermatophytosis of the thighs due to Tr. mentagrophytes which, because of the unusual clinical picture, caused considerable diagnostic and therapeutic problems.

\section{Case report}

A 22 year-old woman was admitted to the outpatient clinic of the Department of Dermatology, Medical University of Lublin, Poland. She presented numerous skin lesions located on the dorsolateral face of the left thigh and the medial surface of the right calf. According to the patient, the initial lesions consisted of small itchy pustules that evolved to exfoliation after scratching. The patient had consulted several physicians and had been prescribed multiple treatments, including local and systemic antifungals (ketoconazole, clotrimazole, isoconazole, terbinafin, ciclopirox olamine), antibiotics (limecycline, metronidazole, clindamycine), antihistamines, and even a short course of steroids, but adherence to therapy was probably poor. Over a six-month period, not only no obvious improvement was noted, but skin lesions had tended to spread. When seen in our department, the patient presented an itchy, edematous, infiltrated, well-circumscribed oval plaque measuring $11 \mathrm{~cm}$ with irregular borders on the lateral and posterior face of the left thigh. The plaque had a bluish-red hue, was overlaid by multiple pink papules and whitish pustules 1-2 $\mathrm{mm}$ in diameter, and was centered by an eschar. On the right thigh, a pink-red irregular patch measuring $2.5 \times 4 \mathrm{~cm}$ was present (Figure 1 ).

Ultrasonographic examination of the skin showed thickening of the dermis with some hypoechogenic foci. Laboratory workup showed normal values for aminotransferases, renal function parameters, electrolytes, protein, bilirubin, glucose, FSH, LH, PRL, total testosterone, CRP and serology tests for all known blood-borne infections and sexually-transmitted diseases. Protein electrophoresis showed no abnormalities. Lymphocytes T and B subpopulations were analyzed by flow cytometry.

Clinical and ultrasonographic examination revealed no abnormalities of the genital tract, although cultures of a vaginal swab yielded Candida glabrata colonies. Due to the lower limb involvement, ultrasonographic examination of peripheral circulation using Doppler mode was performed. Deep veins from the tibio-fibular to the iliac segments were examined.
Neither morphological nor flow changes were detected with a proper Valsalva test. Large and small saphenous veins showed no insufficiency. Lower limbs arteries were unchanged, with proper three-phasic flow spectrum measured on both tibial arteries.

Histopathologic examination of a skin biopsy showed epidermal acanthosis, parakeratotic hyperkeratosis with a scale-crust, possibly an old pustule drying up that was probably related to a hair follicle, although the sections contained no visible hair follicles. The dermis contained a rather dense, diffuse inflammatory infiltrate, tending to aggregate around blood vessels; it consisted mostly of lymphocytes and eosinophils, with extravasated red blood cells. These changes were consistent with dermatophytosis, although the negativity of PAS staining did not allow confirmation of this diagnosis; they were also consistent with insect bites because of the large number of eosinophils and the epidermal changes. Direct microscopic examination with $10 \% \mathrm{KOH}$ was negative; however, skin cultures on BioMerieux media revealed Tr. mentagrophytes (Figures 2, 3, 4).

Following the diagnosis of Trichophyton infection, the patient was treated with a combination of isoconazole nitrate and difluocortolone valerate for one month. After therapy, both direct microscopic mycologic examination and culture on BioMerieux medium were negative; however, the lesions persisted, assuming a completely different aspect. Ultrasonographic examination revealed chronic edema. Cryotherapy with liquid nitrogen was started. This led to a spectacular improvement: the surface of the skin became almost normal, merely showing slight discoloration. The patient did not present any new lesions during the follow-up period, and has remained disease-free for several months (Figure 5).

\section{Discussion}

In the majority of cases, Tr. mentagrophytes infections tend to be confined to a single site on the exposed body area. These sites include primarily the extremities, most frequently the hands and wrists, although tinea corporis, unguium, capitis or kerion and tinea barbae have also been reported $[4,5]$. The infection may have an impact on immunological and inflammatory responses, as phagocytosis has been proven to be compromised [5].

Phagocytosis is the main process responsible for the defense against dermatophytes. Phagocytes released toxic enzymes and kill fungal elements by ingestion and digestion. In one study, phagocytosis was found to be altered in all patients with chronic trichophytosis [6]. There is no consensus as to whether immune responses play a key role in dermatophytosis. Despite an altered phagocyte function has been reported, we found normal immunoglobulin levels and 


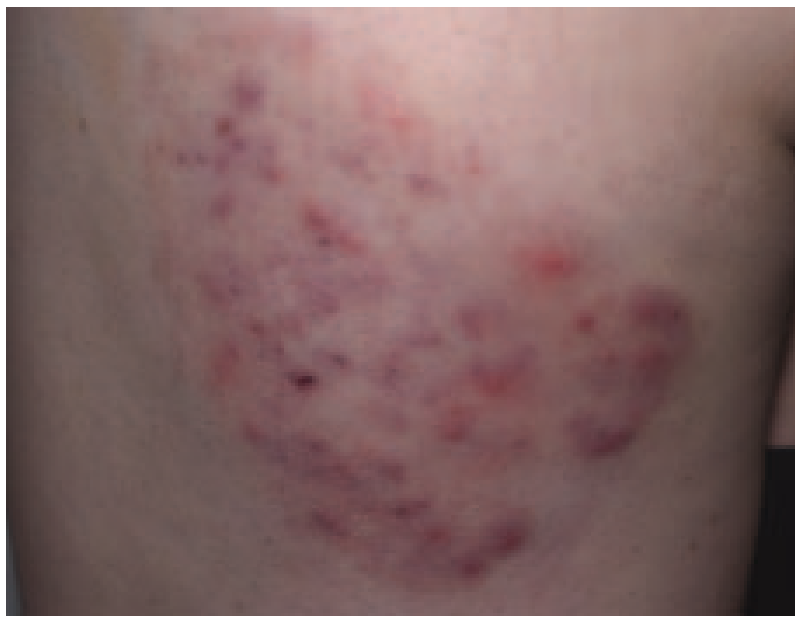

Figure 1. Lesions of the right thigh before treatment

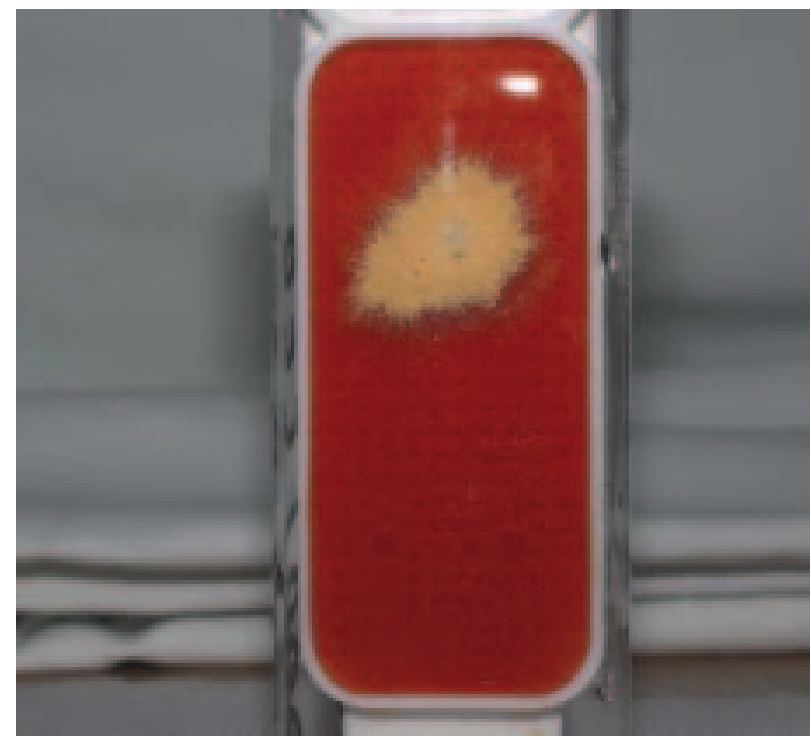

Figure 2. Trichophyton mentagrophytes culture on BioMerieux medium

T- and B-cell count were found. Cellular cytotoxicity was also normal, although not essential in defense against dermatophyte infection. Dermatophytes, mostly Tr. rubrum, possibly produce various mycotoxins, some of which can exert an immunosuppressive activity, favoring the chronicity of infection $[6,7]$.

It has been previously shown that $T r$. mentagrophytes induces interleukin (IL)-8 and tumor necrosis factor (TNF)- $\alpha$ production from keratinocytes, which in turn chemoattract and activate neutrophils. In addition, other cytokines may regulate the immune response by normal human epidermal keratinocytes (NHEK). Dermatophytes could induce cytokine production by NHEK at the transcriptional level, although their ability to induce cytokine production differs among particular species [7].

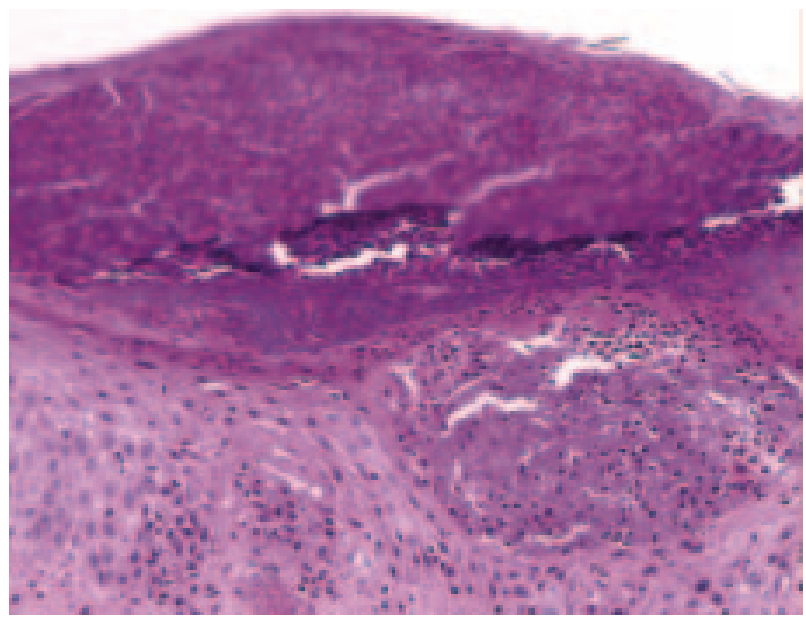

Figure 3. Microscopic picture of Majocchi's granuloma

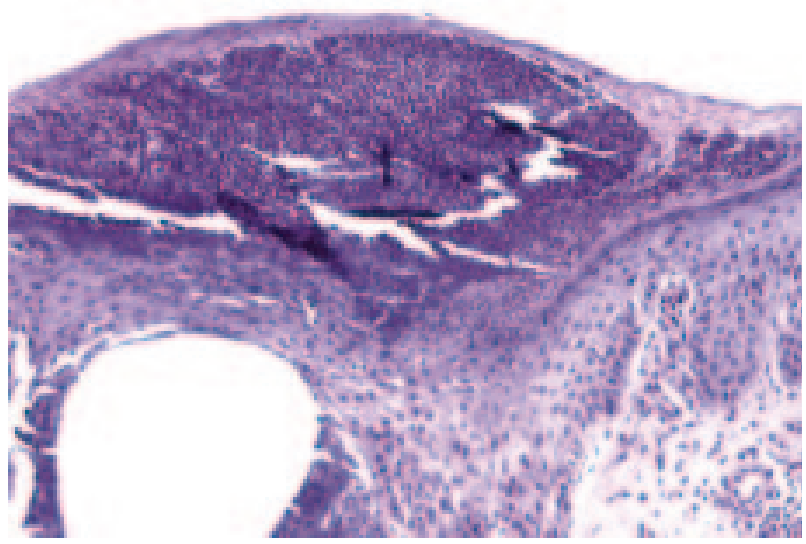

Figure 4. Microscopic aspect of the skin biopsy specimen taken from the right thigh (PAS negative)

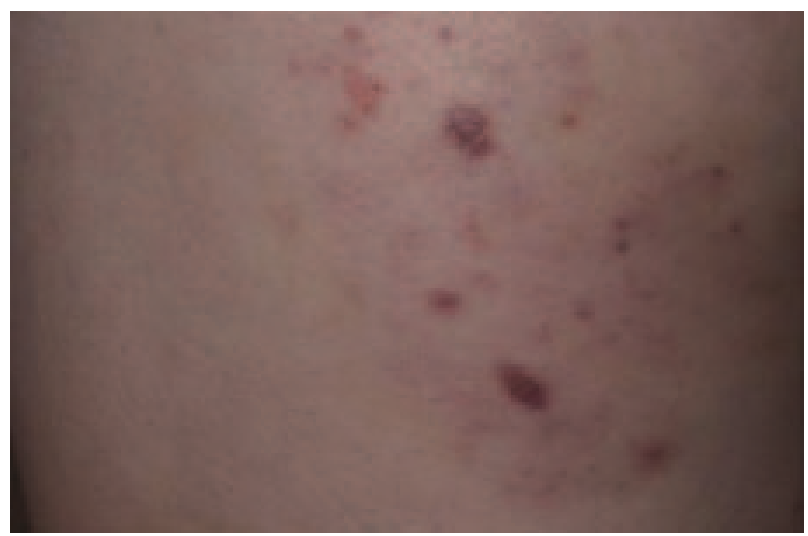

Figure 5. Skin appearance after successful combined therapy with antifungals and cryotherapy

A correct diagnosis is essential for the proper management of dermatophytosis. Skin and nail scrapings and epilated hairs are adequate specimens for both direct examination and culture, provided an ad- 
equate amount of material is obtained. In patients with suspected skin dermatophytosis, local medications should be removed. The lesions should be firmly scraped at the advancing border with a blunt scalpel, tweezers or a curette $[8,9]$.

The lesions present in our patient had a misleading clinical aspect and caused diagnostic difficulties. They can be classified as Majocchi's granuloma (MG). MG or Granuloma trichophyticum, first described in 1883, may develop on any hair-bearing area, most often the scalp, face, forearms, hands, and legs. The fungal infection may be due to, or linked with, widespread contiguous dermatophytosis (following contact with infected animals), use of topical steroids and/or immunosuppression. MG may occasionally mimic Kaposi's sarcoma (especially in patients with AIDS) or lymphocytoma cutis. Our patient had no clinical or laboratory evidence of immunosuppression. The lack of efficacy of the initial treatment might have resulted from an incorrect medication or insufficient treatment duration [10]. Retrospectively, this last hypothesis seems to be more likely, as terbinafin, used in the first phase of treatment, has been reported to be highly effective [11]. Most antifungals exert only fungistatic activity, therefore they only temporarily inhibit fungal growth. If this treatment is too short, dermatophytes and mycotoxins may persist, resulting in recurrence. Some of the newer antifungals, such as terbinafin, have fungicidal and probably immunostimulating activity on neutrophils, accounting for their high efficacy in chronic trichophytosis [12].

Superficial mycoses are not commonly treated with thermotherapy or cryotherapy, probably because they readily respond to standard local or systemic antifungals. However, a case of Malassezia pachydermatis in a dog owner treated with several systemic antimicrobials in addition to liquid nitrogen without relapse has been reported [13]. There are also scattered reports of subcutaneous fungal infections, such as a mycetoma, sporotrichosis, or chromoblastomycosis, responding to cryotherapy. Successful treatment regimens reported include topical ketoconazole combined with applications of liquid nitrogen over an 18-week period or liquid nitrogen monotherapy with either 12 applications over a 29-week period or four applications over an eight-week period [14-16]. The mechanism by which cryotherapy clears chromomycosis is unclear, because fungi are resistant to temperatures lower than those reached with cryotherapy; it is conjectured that fungi are secondarily destroyed as tissue necrosis occurs [17]. Cryotherapy may be an important part of complex treatment, particularly in removing post-infectious problems.

In conclusion, infections with Tr. mentagrophytes may develop on different cutaneous sites. An unusual clinical presentation, and non-responsiveness to treatment, should prompt revision of the primary di- agnosis. A negative result of direct microscopy should not exclude the diagnosis of dermatophytosis but should be checked with cultures, which may prove positive, as in our patient. Proper choice and duration of therapy are essential in treating chronic fungal infections. Cryotherapy should be considered as an adjuvant therapeutic method in cases that do not respond to conventional antifungal medication.

\section{References}

1. Richardson MD, Warnock DW. Fungal Infection: Diagnosis and Management. Blackwell Scientific Publications, London. 1993.

2. Almeida SR. Immunology of Dermatophytosis. Mycopathologia. 2008;166:277-283.

3. Dahl LA. Dermotophytosis and the immune response. $\mathrm{J} A \mathrm{~m}$ Acad Dermatol. 1994;31:S34-41.

4. Wagner DK, Sohnle PG. Cutaneous defenses against dermatophytes and yeasts. Clin Microbiol Rev. 1995;8:317-335.

5. Jones HE. Immune response and host resistance of humans to dermatophyte infection. J Am Acad Dermatol. 1998;28:S12-18.

6. Gregurek-Novak T. Effect of infection with Trichophyton mentagrophytes varietas interdigitale on phagocytosis in humans. J Eur Acad Dermatol Venereol. 2004;18:160-163.

7. Tani K, Adachi M, Nakamura Y et al. The efect of dermatophytes on cytokine production by human keratinocytes. Arch Dermatol Res. 2007;299:381-387.

8. Richard JL. Additional mycotoxins of potential importance to human and animal health. Vet Hum Toxicol. 1990;32:63-69.

9. Rhee DY, Kim MS, Chang SE et al. A case of tinea manuum caused by Trichophyton mentagrophytes var. erinacei: the first isolation in Korea. Mycoses. 2008;52:287-290.

10. Brod C, Benedix F, Rocken M, Schaller M. Trichophytic Majocchi granuloma mimicking Kaposi sarcoma.J Dtsch Dermatol Ges. 2007;5:591-593.

11. Friedlander SF, Aly R, Krafchik B et al. Terbinafine in the treatment of Trichophyton tinea capitis: a randomized, double-blind, parallel-group, duration-finding study. Pediatrics. 2002;109:602-607.

12. Georgopapadakou NH, Walsh TJ. Antifungal Agents: Chemotherapeutic Targets and Immunologic Strategies. Antimicrob Agents Chemother. 1996;40:279-291.

13. Fan YM, Huang WM, Li SF et al. Granulomatous skin infection caused by Malassezia pachydermatis in a dog owner. Arch Dermatol. 2006;142:1181-1184.

14. Pimentel ER, Castro LG, Cuce LC, Sampaio SA. Treatment of chromomycosis by cryosurgery with liquid nitrogen: a report on eleven cases. J Dermatol Surg Oncol.1989;15:72-77

15. Kullavanijaya P, Rojanavanich V. Successful treatment of chromoblastomycosis due to Fonsecaea pedrosoi by the combination of itraconazole and cryotherapy. Int J Dermatol.1995;34:804-807.

16. Bargman H. Successful treatment of cutaneous sporotrichosis with liquid nitrogen: report of three cases. Mycoses. 1995;38:285-287.

17. Doherty CB, Doherty SD, Rosen T. Thermotherapy in dermatologic infections. J Am Acad Dermatol. 2010;62:909-927.

Submitted: 8 January, 2012 Accepted after reviews: 30 January, 2012 